\title{
Giant cemento-ossifying fibroma-A case report and review of literature
}

\section{U. S. Pal, Nimisha Singh, Shailendra Kumar, R. K. Singh, Laxman R. Malkunje}

Department of Oral and Maxillofacial Surgery, K.G. Medical University, Lucknow, India Email: uspalkgmc@yahoo.co.in

Received 11 October 2012; revised 18 November 2012; accepted 29 November 2012

\begin{abstract}
Cemento-ossifying fibroma (COF) of maxilla is comparatively rare lesion of the maxillofacial region. There is often a misdiagnosis in the category of fibroosseous lesions, owing to an overlap of clinical, histological and radiographic features amongst the separate entities. We present a case of giant maxillary COF causing extensive disfiguration of the face, along with extensive review of the clinico-pathologic and treatment aspects of the fibro-osseous lesions.
\end{abstract}

Keywords: Cemento-Ossifying Fibroma; Fibrous Dysplasia; Ossifying Fibroma; Fibro-Osseous Lesion

\section{INTRODUCTION}

Cemento-ossifying fibromas (COF) of maxilla are comparatively rare lesions of the maxillofacial region. There is lot of confusion in diagnostic criteria in particular reference to the category of fibro-osseous lesions, owing to an overlap of clinical, histological and radiographic features amongst the separate entities. We present a case of giant maxillary COF causing extensive disfiguration of the face, managed by surgical excision and prosthetic reconstruction, along with extensive review of the clinicopathologic and treatment aspects of the fibro-osseous lesions.

\section{CASE REPORT}

A 27 year old female visited our outpatient clinic with a huge facial swelling and disfiguration of face which was gradually increasing for last ten years. A painless mass was visible over entire antero-lateral aspect of maxilla, causing distortion of the face, involving eye, nose and upper lip (Figures 1 and 2).

Skin over the swelling was excoriated and ulcerated in the infraorbital region. There was complete and partial obstruction of right and left nares respectively. Vision on the right side was lost due to involvement of the orbit. Comparatively, the palatal surface was less involved. On palpation, infraorbital nerve seemed to be involved, as she presented with hypoaesthesia. A differential diagnosis included fibrous dysplasia, periapical cemento-osseous dysplasia, florid cemento-osseous dysplasia, cementossifying fibroma, cementifying fibroma, ossifying fibroma and calcifying epithelial odontogenic tumour (CEOT).

CT scan findings revealed a bony expansion due to a huge multiloculated mass with varying density (Figure 3).

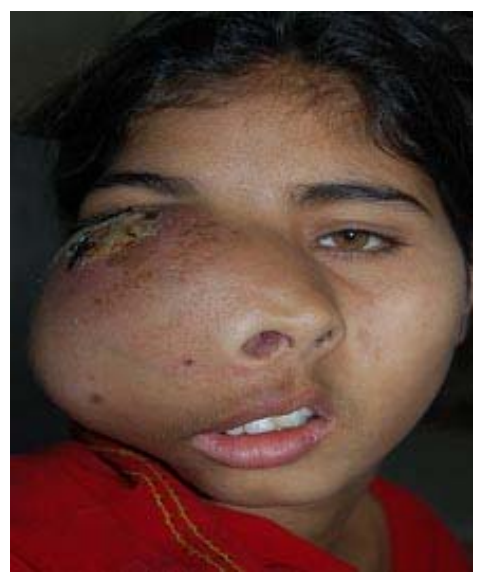

Figure 1. Showing extraoral aspect of tumour.

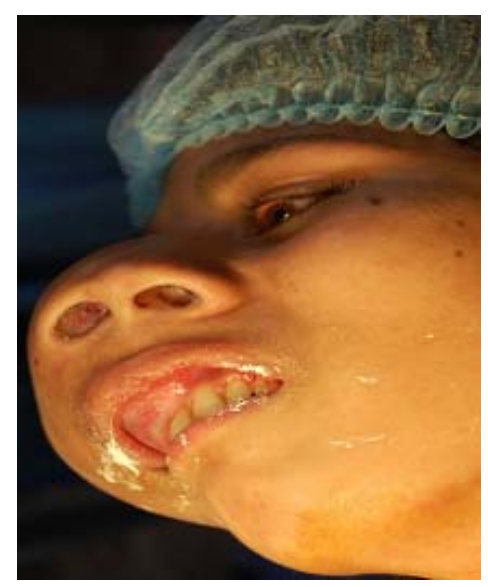

Figure 2. Showing intra-oral aspect of tumor. 


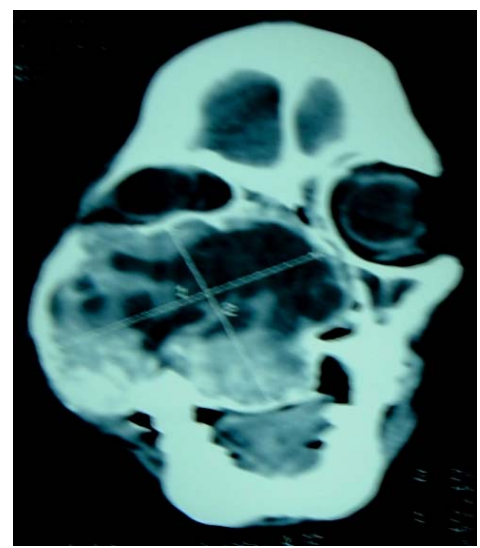

Figure 3. Coronal section of lesion.

The mass had expanded to involve orbital floor, maxillary sinus, nasal septum and palate. Preoperative biopsy reported the lesion as COF.

Surgery was planned under general anesthesia and the lesion was explored through Weber-Fergusson incision. Wide surgical excision (Figure 4) was followed by skin closure (Figure 5).

A palatal prosthesis was made. There was acceptable facial conformation after closure. Since the lesion was huge, we had not done any reconstruction at that time and she was planned for secondary correction after observation for any recurrence. Patient recovered in regular course of time and was discharged from the hospital in satisfactory condition. The final histopathology report was consistent with the previous diagnosis of COF (Figures 6 and 7).

Pathology sample with Hematoxylin and Eosin staining on low and high power fields showed spherical masses with ossification in cellular stroma.

\section{DISCUSSION}

COF manifests as slow growing, asymptomatic, intraosseous mass, more common in women than in men, with female:male ratio of 2:1 [1]. It is common in mandible, $62 \%$ to $89 \%$, about $77 \%$ in the premolar region, age group 20 to 40 years [2]. COF is typically well-circumscribed, solitary radiolucency with scattered radiopaque foci. It maintains a round shape, expands the surrounding cortical bone with no cortical violation, may involve nasal septum, infraorbital foramen, and orbital floor and cause tooth divergence. When COF is present in children it is quite aggressive, hence named juvenile aggressive cemento ossifying fibroma. The common clinical manifestations are proptosis, other visual disturbances with progressive blindness, airway obstruction, and frequent headaches. These lesions have a predilection for supraorbital locations (frontal, ethmoid and maxillary sinus) [3]. The aggressive behavior varies from bowing or pushing of adjacent bone to direct invasion through bone,

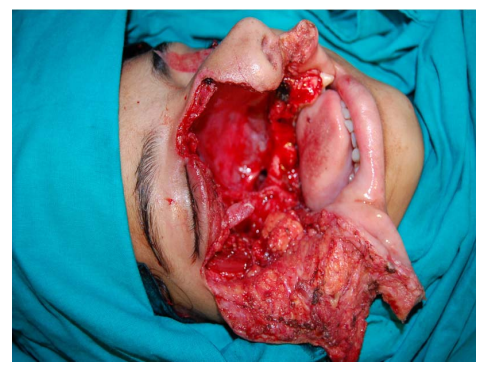

Figure 4. Showing intra-operative picture.

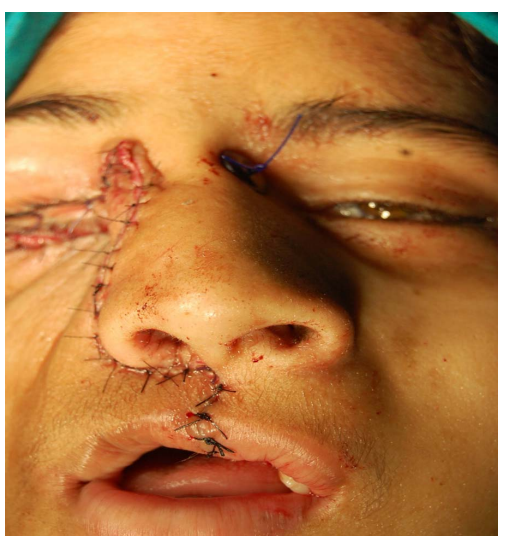

Figure 5. Showing picture after wound closure.

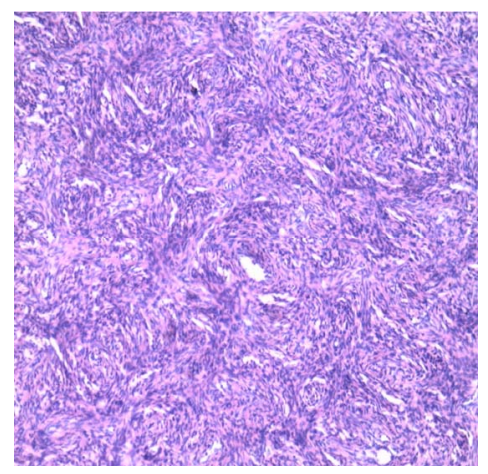

Figure 6. Photomicrograph of lesion in low power.

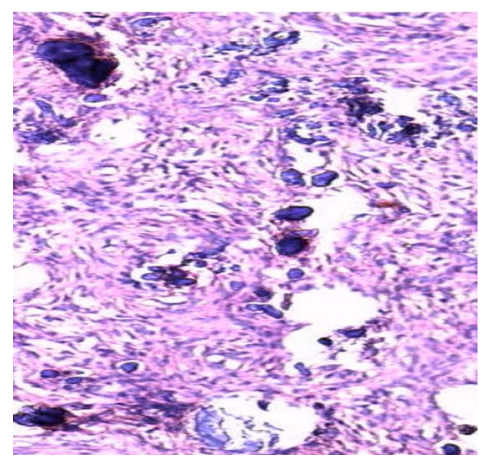

Figure 7. Photomicrograph of lesion in high power. 
with extensive extension into adjacent anatomic compartments involving several sinuses, nasal cavity, nasopharynx, palate, and the cranial cavity. The radiographic features are valuable for diagnosis and determination of the extent of the lesion. With computed tomography, COF may appear as an expansile mass covered by a thick shell of bone density with a multiloculated internal appearance and a varying density content.

COF belongs to the group of fibro-osseous lesions that are histologically benign tumors of the jaws, and present microscopic features like benign connective tissue matrix and trabeculae of new bone [4]. The biologic behavior of these lesions ranges from indolent to aggressive, and inflammatory to neoplastic. The term COF is used for the lesions that arise from periodontal ligament tissue and may contain a mixture of small deposits of bone and cementum. No universally accepted classification exists for these lesions, however Waldron's concept of categorization (Table 1) has been widely used which addresses good clinical and radiological information [5].

Pathologic examination of COF demonstrates irregularly-shaped calcifications within a hypercellular fibrous connective tissue stroma. The appearance of calcifications varies according to the stages of bone and cementum deposition. The spherical ossicles, are concentric or laminated, and include osteocytes, indicating an osteogenic origin. Besides the ossicles, there is a cellular spindle cell stroma that shows a variable mixture of fibrous or myxiod material and giant cell are present.

The differential diagnoses include lesions that contain radiopacities within a rounded radiolucent mass like osteosarcoma or chondrosarcoma, odontogenic cysts, fibrous dysplasia. The factor that differentiates COF from aggressive sarcomas and carcinomas is its well-defined border. Fibrous dysplasia presents a classical "ground glass” appearance. In most cases fibrous dysplasia, diagnosis is made in patients younger than 30 years of age and in many instances, even younger than 20 years. Histologically there is a cellular fibrous connective tissue in which are dispersed, irregular, non-oriented and discontinuous trabeculae of woven bone. The trabeculae may be partially calcified. The bone appears to arise directly from the fibrous stroma and is not rimmed by osteoblasts. Fibrous dysplasia occurs within the maxilla more often than in the mandible [6]. Extension from the maxilla can extend to involve the maxillary sinus, zygoma, sphenoid, and the orbital floor.

Another fibro-osseous lesion, unique to the nasal cavity, paranasal sinuses and orbit, known as "aggressive psammomatiod ossifying fibromas”, “juvenile or young ossifying fibroma" and "juvenile active ossifying fibroma”; tends to affect younger patients [7]. Cases where most of the calcified fragments are immature cementum, with basophilic coloration on hemotoxillin and eosinstained sections, are COF. Cases where the calcified fragments are osteoid, with typical eosinophilic coloration, are ossifying fibroma (OF) [8]. It is believed that $\mathrm{OF}$ and $\mathrm{COF}$ arise from the same progenitor cell; but produce different amounts of bone and cementum within any one lesion. OF presents as a solitary slow-growing tumour. In contrast to fibrous dysplasia, which demonstrates ill-defined or diffuse margin OF has expansile and sharply defined margins, often with a radiolucent peripheral component radiologically. OF is almost exclusively a lesion of the bones of the skull and face, with the mandible involved more often than the maxilla. Patients are usually between 20 to 40 years of age at the time of diagnosis.

Other lesions considered in differential diagnosis are periapical cemento-osseous dysplasia, florid cementoosseous dysplasia and CEOT. Periapical cemento-osseous dysplasia most often involves the apical areas of vital mandibular incisor teeth [9]. It is invariably an asymptomatic lesion that is usually discovered on a radiographic examination. A well circumscribed, radiolucent, mixed lucent to opaque or radiopaque, involving the apices of one or more teeth is observed on radiographic examination. Individual lesions are not often more than $1.0 \mathrm{~cm}$ and most are even smaller than $0.5 \mathrm{~cm}$ in size. The microscope findings are identical with those of focal cemento-osseous dysplasia [10]. The majority of cases occur in the fourth and fifth decades of life. An important distinction from ossifying fibroma is that the dysplastic lesions is gritty, hemorrhagic and is curetted with difficulty. Microscopically the lesion shows a cellular fibrous stroma that is moderately vascular and contains irregular trabeculae of woven bone, cementum like calcifications, or both scattered foci of multinucleated giant cell.

Table 1. Waldron's classification of fibro-osseous lesions.

\begin{tabular}{ll}
\hline 1. Fibrous dysplasia. & $\begin{array}{l}\text { A. Polyostotic. } \\
\text { B. Monostotic. }\end{array}$ \\
$\begin{array}{ll}\text { 2. Reactive (presumably of periodontal ligament origin) lesion. } \\
\text { A. Periapicalcemento-osseous dysplasia. }\end{array}$ & $\begin{array}{l}\text { B. Focal cemento-osseous dysplasia. } \\
\text { C. Florid cemento-osseous dysplasia. }\end{array}$ \\
3. Putative fibro-osseous neoplasms. & $\begin{array}{l}\text { A. Cementifyingfibroma. } \\
\text { B. Ossifying fibroma. } \\
\text { C. Cemento-ossifying fibroma. }\end{array}$ \\
\hline
\end{tabular}


Florid cemento-osseous dysplasia is a lesion seen almost exclusively in middle aged and older women [5]. There is strong tendency towards a bilateral, symmetric involvement and it is not uncommon to find extensive lesions in all posterior quadrants of the jaw. Microscopically, the lesions show an admixture of woven bone trabeculae and cementum like calcifications in a fibroblastic stroma.

Calcifying epithelial odontogenic tumor is tumour found more commonly in mandible than maxilla in 4th 5th decade of life. Clinically it is slow growing painless mass. It appears as radiolucent area with small radiopacties within. Histologically there are deposits of amyloid like material throughout epithelium and connective tissue of the lesion.

Complete surgical excision, when possible, it is the treatment of choice [10]. This may not always be feasible depending on the location and extent of the tumour. Radiotherapy is contraindicated because of its radio-resistance and post-radiation complications. Despite the tendency for local invasive growth and recurrence, it is not reported to metastasize. Regular follow ups are recommended.

\section{CONCLUSION}

In conclusion, the variability of fibro-osseous lesions are so great that can present markedly separate clinical and radiologic entities, depending on the proportion of fibrous to osseous tissue within the lesion. Historical, clinical, radiographic and histopathologic data combined with surgical findings are though essential, but still causing a lot of confusion in diagnosis and treatment pattern. Probably it requires further studies to determine the exact nature of these lesions, to pin point the characteristics of its clinical features, radiological and histological features for a definitive diagnosis and treatment planning.

\section{REFERENCES}

[1] More, C., Thakkar, K. and Asrani, M. (2011) Cemento- ossifying fibroma. Indian Journal of Dental Research, 22, 352-355. doi:10.4103/0970-9290.84296

[2] Hekmatnia, A., Ghazavi, A., Saboori, M., Mahzouni, P., Tayari, N. and Hekmatnia, F. (2011) A case report of cemento-ossifying fibroma presenting as a mass of the ethmoid sinus. Journal of Research in Medical Science, 16, 224-228.

[3] Brademann, G., Werner, J.A., Jänig, U., Mehdorn, H.M. and Rudert, H. (1997) Cemento-ossifying of the premastoid region: Case report and review of the literature. Journal of Laryngology \& Otology, 111, 152-155. doi:10.1017/S0022215100136709

[4] Singhal, A., Ram, R., Singhal, P., Bhatnagar, S. and Das, U.M. (2011) Cemento-ossifying fibroma of maxillary antrum in a young female patient. Journal of Indian Society of Pedodontics and Preventive Dentistry, 29, 44-47. doi:10.4103/0970-4388.90740

[5] Waldron, C.A. (1993) Fibroma-osseous lesions of the jaws. Journal of Oral and Maxillofacial Surgery, 51, 828835. doi:10.1016/S0278-2391(10)80097-7

[6] Lello, G.C. and Sparrow, O.C. (1985) Craniofacial polyostotic fibrous dysplasia. Journal of Oral and Maxillofacial Surgery, 13, 267-272.

[7] Malathi, N., Radhika, T., Thamizhchelvan, H., Ravindran, C., Ramkumar, S., Giri, G.V.V. and Gopal, D. (2011) Psammomatoid juvenile ossifying fibroma of the jaws. Journal of Oral and Maxillofacial Pathology, 15, 326329.

[8] Eversole, L.R., Leider, A.S. and Nelson, K. (1985) Ossifying fibroma: A clinicopathologic study of 64 cases. Oral Surgery, Oral Medicine, Oral Pathology, 60, 505511. doi:10.1016/0030-4220(85)90239-7

[9] Slootweg, P.J. and Muller, H. (1990) Differential diagnosis of fibro-osseous jaw lesions: A histogical investigastion of 30 cases. Journal of Cranio-Maxillofacial Surgery, 18, 210-214. doi:10.1016/S1010-5182(05)80413-5

[10] Cheng, X.B., Li, Y.P., Lei, D.L., Li, X.D. and Tian, L. (2011) Surgical resection of a huge cemento-ossifying fibroma in skull base by intraoral approach. Journal of Craniofacial Surgery, 22, 594-597. doi:10.1097/SCS.0b013e31820745ff 